\title{
Brote de dermatofilosis en bovinos del altiplano de Jujuy, Argentina
}

\author{
Marin, R.E. ${ }^{\text {; Saez, J.2; Saez, V. }}{ }^{2}$ \\ ${ }^{1}$ Facultad de Ciencias Agrarias, UNJu, Alberdi 47, 4600-Jujuy, Argentina. \\ ${ }^{2}$ Lab.Vetlab, Independencia 920, Jujuy. E-mail: raulemarin@hotmail.com
}

\begin{abstract}
Resumen
Marin, R.E.; Saez, J.; Saez, V.: Brote de dermatofilosis en bovinos del altiplano de Jujuy, Argentina. Rev. vet. 29: 2, 139-141, 2018. Se describe un brote de dermatofilosis (Dermatophilus congolensis) en bovinos de la puna de Jujuy. El cuadro se presentó en un rodeo bovino criollo nativo, durante la estación lluviosa en el verano climático de la región. Los animales afectados presentaron dermatitis exudativa proliferativa no pruriginosa con formación de costras, afectando el espacio interdigital de los cuatro miembros, y en forma severa y localmente extensiva en los miembros posteriores y el interior de las fosas nasales. El diagnóstico se realizó en base a la presentación clínica, la identificación del agente causal en frotis obtenido del material dérmico, y las características epidemiológicas, considerando que los factores climáticos y las condiciones de manejo favorecieron la presentación de la enfermedad.
\end{abstract}

Palabras clave: bovinos, Dermatophilus congolensis, altiplano, Jujuy, Argentina.

\begin{abstract}
Marin R.E.; Saez J.; Saez V.: Outbreack of dermatophilosis in cattle of the Puna region of Jujuy, Argentina. Rev. vet. 29: 2, 139-141, 2018. An outbreak of dermatophylosis (Dermatophilus congolensis) in cattle from the Puna region at Jujuy province, is described. The disease was present in a native creole bovine herd during the rainy season (summer). The affected animals presented symmetrical and non-pruritic proliferative exudative dermatitis with crust formation, affecting the interdigital space of the four limbs, and severe and locally extensive form in the hind limbs and in the interior of the nostrils. The diagnosis was performed based on the clinical presentation and the identification of the causative agent in smears obtained from crust material as well as the epidemiological characteristics, considering that both climatic factors and management conditions favored the presentation of the disease.
\end{abstract}

Key words: cattle, Dermatophilus congolensis, puna region, Jujuy, Argentine.

\section{INTRODUCCIÓN}

La dermatofilosis es una dermatitis superficial no pruriginosa, proliferativa y exudativa con producción de costras, que puede presentar curso agudo, subagudo o crónico, causada por Dermatophilus congolensis, un actinomiceto gram positivo, que produce zoosporas móviles. Es frecuente en bovinos, ovinos y caprinos, aunque afecta a una gran variedad de especies, incluida el hombre ${ }^{1-5,9}$.

Es una enfermedad infecto-contagiosa y su aparición está condicionada a la presencia de animales portadores. Se transmite por un agente oportunista que reside normalmente en la piel, pudiendo colonizarla e invadirla bajo condiciones ambientales favorables y situaciones estresantes para el animal ${ }^{2}$. Su presentación es más común en los trópicos y subtrópicos cálidos, y en áreas con precipitaciones pluviales altas.

Recibido: 8 febrero 2018 / Aceptado: 18 julio 2018

Presentado XX Reun.AAVLD, Tucumán, Argentina, 2014
La enfermedad fue descripta por primera vez por Vonsoceghem (1915) en bovinos en el Congo Belga (citado por Shoorijeh et al., 2008). Es una enfermedad de distribución mundial ${ }^{1,2,6}$, aunque en Argentina ha sido escasamente reportada ${ }^{7,8}$.

En la presente comunicación se describe un caso de dermatofilosis en bovinos del altiplano jujeño de Argentina.

\section{MATERIAL Y MÉTODOS}

En febrero de 2014 se inspeccionó un rodeo de 50 bovinos raza criolla nativa, que presentaba animales con dermatopatía severa, pertenecientes a un sistema de ganadería familiar, ubicado en la localidad de Puesto del Marqués, Departamento Cochinoca. (2232“42”S-6541"45”O), a $3502 \mathrm{msnm}$, en la región puna de la Provincia de Jujuy.

Se realizó inspección clínica y toma de muestras de costras dérmicas con pelos e hisopado vigoroso de la mucosa nasal. Las muestras fueron depositadas en tu- 
bos de ensayo conteniendo solución fisiológica estéril, hasta lograr el reblandecimiento de las mismas. Luego fueron colocadas en portaobjetos, dejándolas secar durante 10 minutos y luego fijándolas con calor y tinción Giemsa $1 / 10$ por $30 \mathrm{~min}$. Posteriormente se observaron al microscopio óptico con 100x.

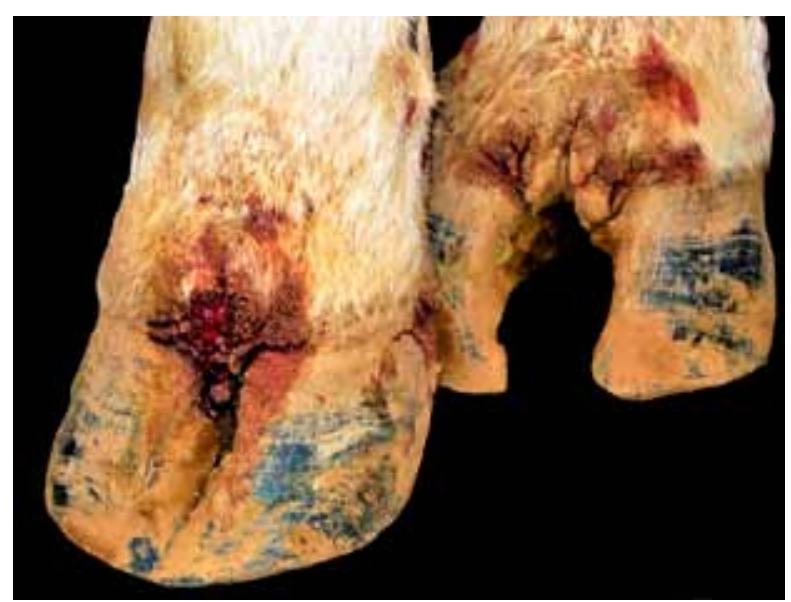

Figura 1. Dermatitis exudativa interdigital.

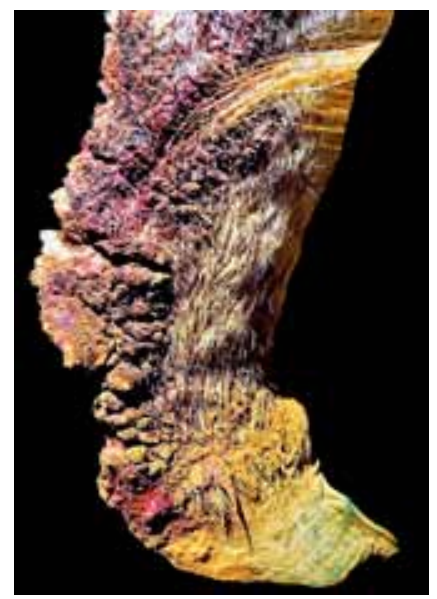

Figura 2. Dermatitis proliferativa exudativa severa.

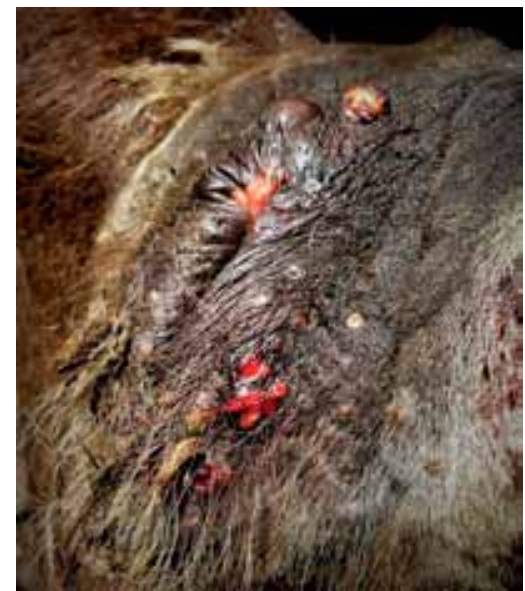

Figura 3. Dermatitis nodular a coalescente en labios vulvares.

\section{RESULTADOS}

La inspección general del rodeo determinó la presencia de dos animales (2/50) de 2 y 4 años aproximadamente, ambas hembras en lactancia con ternero al pie, que presentaban dermatitis exudativa simétrica no pruriginosa severa, afectando el espacio interdigital de

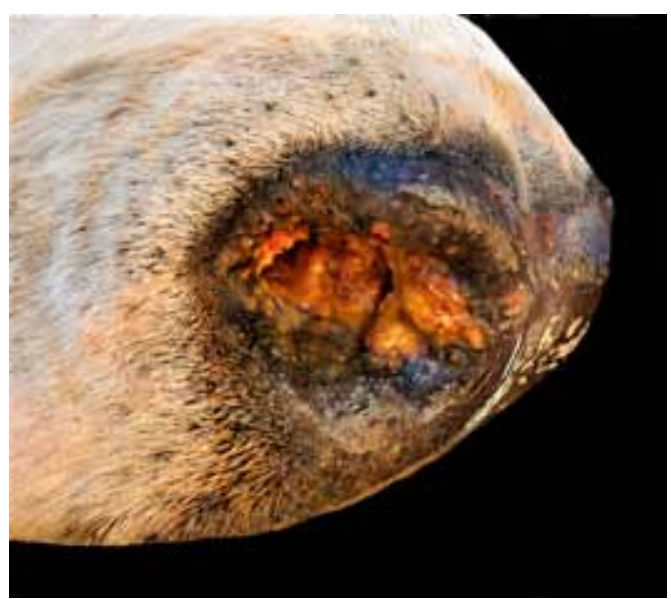

Figura 4. Dermatitis intranasal de aspecto nodular a coalescente.

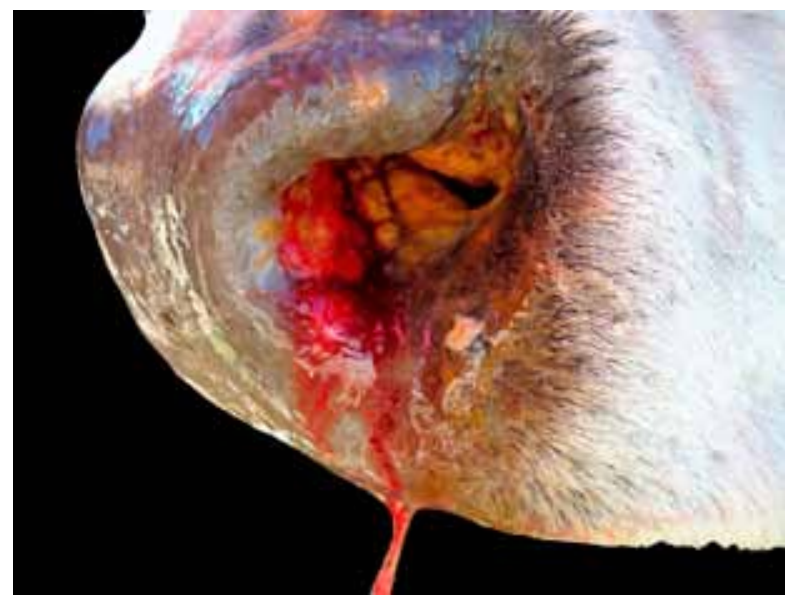

Figura 5. Dermatitis intranasal de aspecto nodular a coalescente, de fácil sangrado.

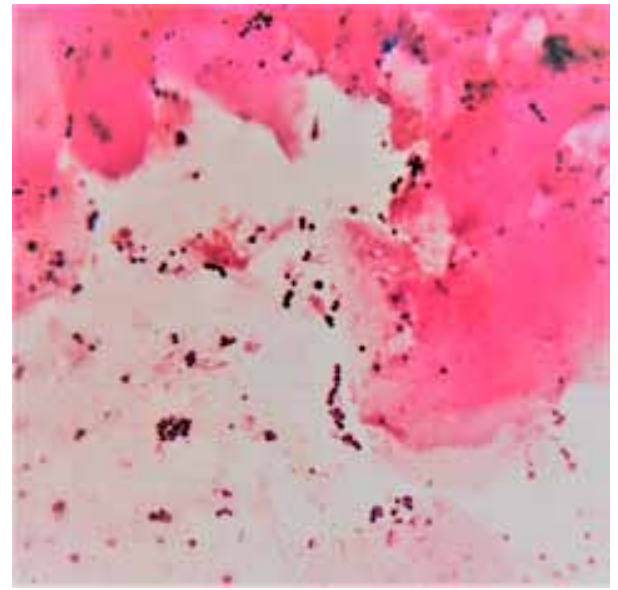

Figura 6. Abundantes formas cocoides en material queratósico. 
los cuatro miembros (Figura 1) y en forma proliferativa localmente extensiva y severa, en la cara posterior de los miembros traseros, desde las pezuñas hasta la región perineal (Figura 2) e inclusive labios vulvares (Figura 3).

Las lesiones dérmicas presentaban aspecto nodular a coalescente proliferativo exudativo, con costras y de fácil sangrado a la palpación con presión leve. En el interior de ambas fosas nasales se observaron lesiones de aspecto nodular a coalescente, de color amarillento y de fácil sangrado (Figura 4 y 5).

La observación microscópica del material costroso demostró la presencia de abundantes formas cocoides esféricas u ovoides, con pequeñas disposiciones de $4 \mathrm{o}$ 5 cocos en fila, junto a material queratósico (Figura 6).

Los animales recibieron antibioticoterapia con oxitetraciclina (larga acción), en dosis de $10 \mathrm{mg} / \mathrm{kg}$ cada 3 días, con resultado satisfactorio luego de 4 aplicaciones.

\section{DISCUSIÓN}

La dermatofilosis ocurre en todo el mundo y puede ser epizoótica en áreas tropicales o subtropicales ${ }^{1} . D$. congolensis es un actinomyceto gram positivo, anaerobio facultativo. Su hábitat natural es desconocido y los intentos por aislarlo del suelo han fracasado. Su proliferación intradérmica es favorecida por la presencia de abrasiones en la piel debido a su poca capacidad de invadir la piel sana, no habiendo podido reproducirse experimentalmente, inclusive con altas dosis infectantes ${ }^{6}$.

Debido a ello se considera que su presentación depende de una variedad de factores predisponentes, principalmente aquellos ambientales y de manejo, en situaciones con elevadas precipitaciones y humedad propias de los climas tropicales y subtropicales, que a la vez también permiten la proliferación de garrapatas, consideradas asociadas a la presentación de estos cua$\operatorname{dros}^{2,5,6}$.

En zonas templadas la enfermedad tiene alta incidencia en las estaciones lluviosas, siendo la humedad el principal factor de riesgo asociado a la presentación de brotes en los rodeos y severa afección individual ${ }^{1,2}$. En la región puna las precipitaciones son escasas y oscilan entre los 200 y $300 \mathrm{~mm}$ anuales, con estación lluviosa exclusivamente en los meses del verano climático, de diciembre a marzo principalmente.

El rodeo problema fue mantenido en pastaje en potreros anegados durante parte del verano lluvioso, lo cual se considera como factor determinante del brote. La permanencia sobre el agua posiblemente favoreció la emulsificación y maceración de la piel, vulnerando su capacidad de barrera y permitiendo la invasión de $D$. congolensis, coincidiendo el lugar de las lesiones con las zonas que presumiblemente estuvieron bajo agua.

Las lesiones dérmicas observadas son coincidentes con las descriptas en la literatura ${ }^{1-3,5,6}$, a diferen- cia de las presentes en la cavidad nasal, las cuales se ubicaban únicamente en la mucosa, sin afectar la piel de los ollares ni otra región circundante, lo cual quizás esté asociado a la menor defensa de un epitelio de mucosas respecto de los otros, así como a la menor ventilación de las fosas nasales manteniendo su humedad debido al hábito alimentario sobre un terreno anegado, con dicha zona semisumergida en el agua durante el consumo.

Los dos animales afectados tenían pobre condición corporal y eran hembras en lactancia con terneros al pie, situación que podría haber actuado como un factor de estrés favoreciendo las bajas defensas ${ }^{2,5,6}$. Los antecedentes de manejo de pastoreo sobre un terreno anegado, la presentación clínica y la identificación de formas compatibles con el agente a partir de las muestras dérmicas, se consideraron suficientes para la conclusión diagnóstica, debido a la morfología del actinomiceto causal, aunque a veces a causa de la preparación vigorosa del frotis, dichas características puedan alterarse ${ }^{1}$.

Otros procedimientos diagnósticos como biopsia de piel para estudios histopatológicos y cultivo microbiológico no pudieron realizarse debido a los costos significativos para un productor de escala familiar.

\section{REFERENCIAS}

1. Anónimo. 2004. Manual de la OIE sobre animales terrestres, capítulo 2.3.10, dermatofilosis, p. 562-563. https:// www.oie.int/doc/ged/D6508.PDF

2. Brayer PD, Meireles MC. 2007. Dermatofilose. En: Doenças de Ruminantes e Eqüideos (Riet-Correa F., Ed.), $3^{\circ}$ ed., Fenovi Editora, São Paulo, Brazil, 573 p.

3. Cattáneo M et al. 2009. Brote de dermatofilosis en terneros. Analecta Vet 29: 32-34.

4. Cunha PH et al. 2009. Dermatofilose: relato de caso em bovinos da raçanelore criados em regime de confinamento. Anais do VIII Congresso Brasileiro de Buiatria, Belo Horizonte, Brazil, p. 88-92.

5. Gebreyohannes M, Gebresselassie M. 2013. An overview on dermatophilosis of animals: a review. J Anim Sci Adv 3: 337-344.

6. Mauldin EA, Peters KJ. 2016. Integumentary System. In: Jubb, Kennedy, and Palmer's Pathology of Domestic Animals (Maxie M.G. ed.), Vol. 1, $6^{\circ}$ ed., Elsevier, Amsterdam, p. 509-736.

7. Otero MM et al. 2003. Ocurrencia de dermatofilosis caprina en la Provincia de Buenos Aires. Analecta Vet 23: 15-18.

8. Pérez CE, Di-Rocco M. 1962. Primer aislamiento efectuado en la República Argentina del Dermatophilus dermatonomus, de lanas de ovinos enfermos de "rasquilla" (¿dermitis contagiosa?). Rev Inv gan 15: 249-255.

9. Shoorijeh J, Badiee S, Bahzadi AM, Tamadon A. 2008. First report of dermatophilosis dermatitis in dairy cows in Shiraz, Southern Iran. Iran J Vet Res 9: 281-282. 